\title{
Tests of product and additive scaling axioms'
}

\author{
ROBERT F. FAGOT ${ }^{2}$ AND MANARD STEWART \\ UNIVERSITY OF OREGON
}

\begin{abstract}
A product axiom for ratio judgments and an additive axiom for difference judgments were tested for six Ss judging brightness. The product axiom was rejected for all six $S$ s whereas the additive axiom was accepted for two Ss. Power functions did not fit the data well, although somewhat better for difference judgments than ratio judgments. A two-stage model fitted somewhat better, but failed to satisfy one important implication of that model, previously confirmed for lifted weights. The data gave stronger support for a theory of brightness estimation based on an additive axiom for difference judgments than a product axiom based on ratio judgments.
\end{abstract}

The methods of ratio and magnitude estimation have been widely used to obtain direct measurements of psychological attributes, but until recently little attention had been paid to making explicit and testing the assumptions on which the methods are based. Steps in this direction have been taken, e.g., by Mashhour (1961), Sjoberg (1965), Svenson and A kesson (1966, 1967), and Poulton (1968).

It seems reasonable that important necessary conditions for a model of ratio and magnitude estimation ought to be thoroughly explored before formal axiomatizations are attempted. In this paper we shall test two such conditions: a product axiom for ratio estimation scaling, and an additive axiom for ratio estimation of differences. The attribute studied will be brightness.

Let $\Psi_{\mathbf{i}}$ denote the psychological magnitude of Stimulus i and define $\Psi_{i j}=\Psi_{i} / \Psi_{j}$. Then the product axiom may be formulated as follows:

Product axiom. For all triples of stimuli, i, j, k.

$$
\Psi_{\mathrm{ij}} \Psi_{\mathrm{jk}}=\Psi_{\mathrm{ik}} \text {. }
$$

For convenience in testing, the product axiom may be written in its logarithmic form:

$$
\log \Psi_{i j}+\log \Psi_{j k}=\log \Psi_{i k}
$$

The product axiom is formulated entirely in terms of observables (numerical responses) and is directly testable. Stimuli are judged in pairs, and hence the axiom is applicable to ratio estimation but not directly to magnitude estimation. The axiom is identical to the condition of "multiplicative transitivity" formulated by Svenson and A kesson (1966), and equivalent to the test for ratio estimation advocated by Eisler (1960).

Beck (1967) applied the method of ratio estimation to the judgment of loudness intervals. For the development of a theory based on such difference judgments, we formulate an additive axiom. Let $\Psi_{j-i}=\Psi_{j}-\Psi_{i}$ denote the psychological magnitude of a directly estimated difference. Then the additive axiom is:

Additive axiom. For all triples of stimuli, i, j, k.

$$
\Psi_{k-j}+\Psi_{j-i}=\Psi_{k-i}
$$

A special feature of these axioms is that they are applied before any scale is constructed, and a subset of the data is used to predict the residual data. Tests based on scales constructed from all the data are more likely to obscure possible violations of particular axioms; i.e., a rejection of a model based on all the data gives less information on the precise nature of the violations.

\section{Subjects}

\section{METHOD}

Subjects were two male and four female undergraduates at the University of Oregon, and were paid $\$ 1.50$ per hour for their time.

\section{Apparatus}

The apparatus, described in detail elsewhere (Eskildsen, 1963), provided four luminous white circular targets $13 \mathrm{~mm}$ in diam, placed horizontally and separated by $90 \mathrm{~mm}$, center to center. The apparatus was located in a light-tight visual tunnel, and Ss were seated at a distance of $12 \mathrm{ft}$ from the targets in an ordinary classroom desk seat. $\mathrm{S}$ reported his estimations orally. A brief dark-adaptation period of $15 \mathrm{~min}$ preceded each experimental session.

\section{Procedure}

Three of the Ss (randomly selected) did the difference estimation task first and the ratio task second, and the other three Ss performed the tasks in reverse order.

Difference estimation task. Each trial began with the presentation of the standard pair, which consisted of two adjacent lights, one set at 30 (always on the left) and the other at $100 \mathrm{ft}-\mathrm{L}$. By pushing a button $\mathrm{S}$ could then move a shutter to view the comparison pair which was always to the left of the standard pair. The $S$ was told to call the difference in brightness for the standard pair 100, and to assign a number to the difference in brightness for the comparison pair in accord with usual magnitude estimation instructions. The $S$ could move the shutter back and forth as many times as he wished to compare the two pairs. The trial ended when $S$ responded and E turned out the lights. The following instructions were read for the difference estimation task:

"In this experiment your task will be to judge the brightness of light, and in particular to judge the difference in brightness of two lights, relative to the difference of two other lights. On each trial you will be presented with two pairs of lights. The first pair will be called the standard pair, and will remain the same throughout the experiment, and will always occur first on every trial. To view the second pair-called the comparison pair-you must push the button attached to your chair. You are to call the difference in brightness for the standard pair 100. Your task is to judge the difference in brightness for the comparison pair of lights relative to the difference in brightness for the standard pair. That is, the number assigned to the difference in brightness for the comparison pair is to be proportional to the difference in brightness for the standard pair. For example, if the difference for the comparison pair seems to be about five times as much as for the standard pair, you are to label the second pair 500, since the standard pair was assigned 100 . On the other hand, should the difference appear to be only one-half that of the standard pair, you are to label the second pair 50. You will then proceed to the next trial. You may move the shutter back and forth to compare the two pairs of lights as often as you wish before making a judgment. Any questions?"

The standard pair for the difference task was, in $\mathrm{ft}-\mathrm{L}, 30$, 
100. The 36 comparison pairs were constructed by taking all possible pair comparisons for the following nine stimuli: 0.1 , $1.5,8,25,60,120,220,380$, and $600 \mathrm{ft}-\mathrm{L}$. The stimuli were selected to give a wide range for $\Psi_{k-i}$ assuming a power law exponent of .25. Analysis of the data showed that the standard pair tended to be judged at the lower end of the distribution of differences, meaning that in most cases Ss were making multiple rather than fractional judgments of differences.

Each $S$ was given 72 trials per day (each day's session lasting about $1 \mathrm{~h}$ ) for five days. These trials consisted of two replications of the set of 36 comparison pairs. Within each replication the order of the pairs was randomized separately for each replication and each day, with the restriction that the last pair of the first replication could not be the same as the first pair of the second. The same random sequence of comparison pairs was used for all six Ss. Thus each $S$ in the course of five days gave 10 magnitude estimations for each comparison pair.

Ratio task. Each trial consisted of the presentation of a single pair of lights, with left and right position randomly assigned. The $S$ made the assignments to both standard and comparison stimulus. Each $S$ was given the following instructions:

"On each trial of the experiment you will be presented a pair of lights. The light on your left will be called the standard, and you should report the apparent brightness of the standard by assigning any number to it in such a way as to reflect its brightness. After you have assigned a number to the standard, you are to estimate the brightness of the right-hand light in relation to the standard. For example, if you had assigned 100 to the standard and the right-hand light appears half as bright as the standard, you should assign 50 to the right-hand light. On the other hand, if the right-hand light appears twice as bright as the standard, the appropriate response would be 200 . Feel free to use any number you think is appropriate for the standard, and be sure you then judge the right-hand light in relation to the standard. Upon the completion of a trial press the button, which will be a signal to me to start the next trial. Please bear in mind that the pairs of lights will vary from trial to trial. Remember, you are to make your judgments only in terms of the brightness of the lights and not in terms of any other properties. Any questions?"

In order to obtain a wide range of $\Psi_{\mathrm{i}} / \Psi_{\mathrm{k}}$ values several sets of triples $\left(\Phi_{1}, \Phi_{j}, \Phi_{k}\right)$ were constructed such that a range of $\Psi_{\mathrm{i}} / \Psi_{\mathrm{k}}$ of about 2 to 10 would be generated under the assumption that a simple power law held for several selected values of the exponent $(\mathrm{k})$. For all triples a random assignment from three stimuli in the middle range was made for $\Phi_{j}$, which guarded against mostly equal values of $\Phi_{i} / \Phi_{j}$ and $\Phi_{j} / \Phi_{k}$ and against extreme values of either. Each triple then provided three pairs of presentation stimuli $\left(\Phi_{\mathrm{i}}, \Phi_{\mathrm{j}}\right),\left(\Phi_{\mathrm{j}}, \Phi_{\mathrm{k}}\right)$, and $\left(\Phi_{\mathrm{j}}\right.$, $\Phi_{k}$ ) for a total of 27 pairs in each set. The first session was then used to obtain an estimate of S's exponent (using ratio estimations, as in the main part of the experiment), and on the basis of this estimate, a particular set of stimuli was selected for a given $S$. On the basis of the first day's estimation of $k$, two Ss were assigned to each of the sets $\mathrm{k}=.40, .35$, and one $\mathrm{S}$ to each of the sets $\mathrm{k}=.30, .25$; i.e., four different blocks of 27 pairs of stimuli were used for a total of six Ss.

The order of the 27 pairs was randomized for each of the four blocks, and the appropriate block was presented to each $S$ a total of 10 times in four days (each day's session lasting about $1 \mathrm{~h}$ ) as follows: Days 2 and 3-two blocks or 54 trials each day; Days 4 and 5-three blocks or 81 trials each day.

\section{RESULTS AND DISCUSSION}

The main analysis was concerned with a test of the product axiom (Eq. 2) and the additive axiom (Eq. 3). In both cases the analysis was concerned with the fit of data points to a theoretical straight line with unit slope and zero intercept.

The data for the test of the product axiom consisted (for each S) of 10 observations for each of the 27 pairs of stimuli presented, with $S$ judging the ratio of brightness between the members of each pair by first assigning a number to the stimulus on the left, then assigning a number to the stimulus on the right relative to the left stimulus. The stimuli were organized into nine independent triples and each triple provided a data point for a test of the product axiom. Thus, a triple of stimuli $\Phi_{i}, \Phi_{j}, \Phi_{k}$ generates a "direct" estimate of $\log \Psi_{i \mathrm{k}}\left(\log \hat{\Psi}_{\mathrm{ik}}\right)$ and an "indirect" estimate $\log \hat{\Psi}^{\prime}{ }_{\mathrm{ik}}=\log \hat{\Psi}_{\mathrm{ij}}$ $+\log \dot{\psi}_{j \mathbf{k}}$.

Figure 1 shows a plot of the mean indirect estimates as a function of mean direct estimates for each of the six Ss. The straight line with unit slope and zero intercept is the theoretical line dictated by the product axiom.

The data for the test of the additive axiom consisted of 10 estimations of each of 36 comparison pairs. Although these 36 pairs provide 84 triples (each triple providing a data point for a test of the additive axiom), not all 84 triples are independent. We mean here by independence that no pair should appear in more than one triple, which guarantees that no two triples in the analysis depend even partially on the same data. (This condition was automatically satisfied by the triples in the product data.) Unfortunately, there are several sets of independent triples. The set used in the analysis was selected as follows. For any triple $\Phi_{i}, \Phi_{j}, \Phi_{k}$, for $\mathrm{i}<\mathrm{j}<\mathrm{k}$ and $\Phi_{i}<\Phi_{j}<\Phi_{k}$, let the "level" of the triple be defined as $k-i$. Then the selection criteria were: (a) The subset should contain
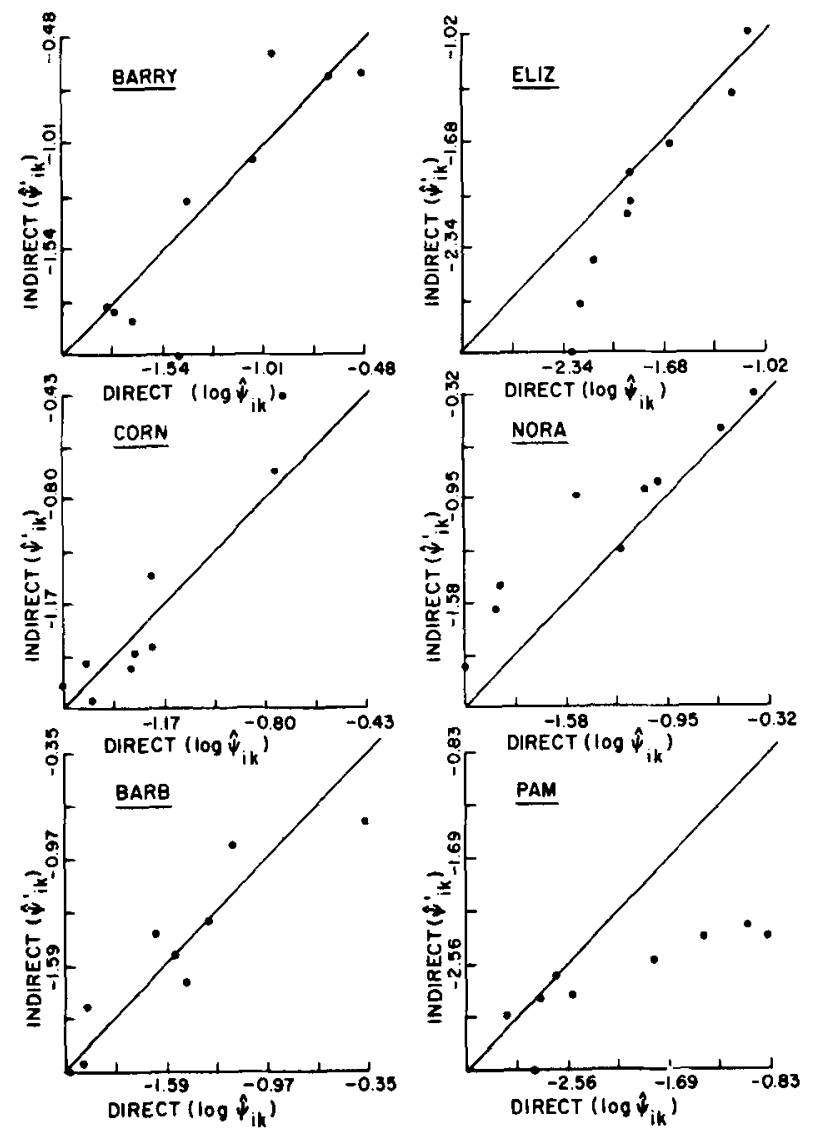

Fig. 1. Test of product axiom: Plots of mean indirect estimates $\left(\log \dot{\Psi}_{i k}^{\prime}=\log \dot{\Psi}_{i j}+\log \dot{\Psi}_{j \mathbf{k}}\right)$ as a function of mean direct estimates $\left(\log \hat{\Psi}_{i k}\right.$ ) based on independent triples. Ss on left did ratio task first and Ss on right did difference task first. 

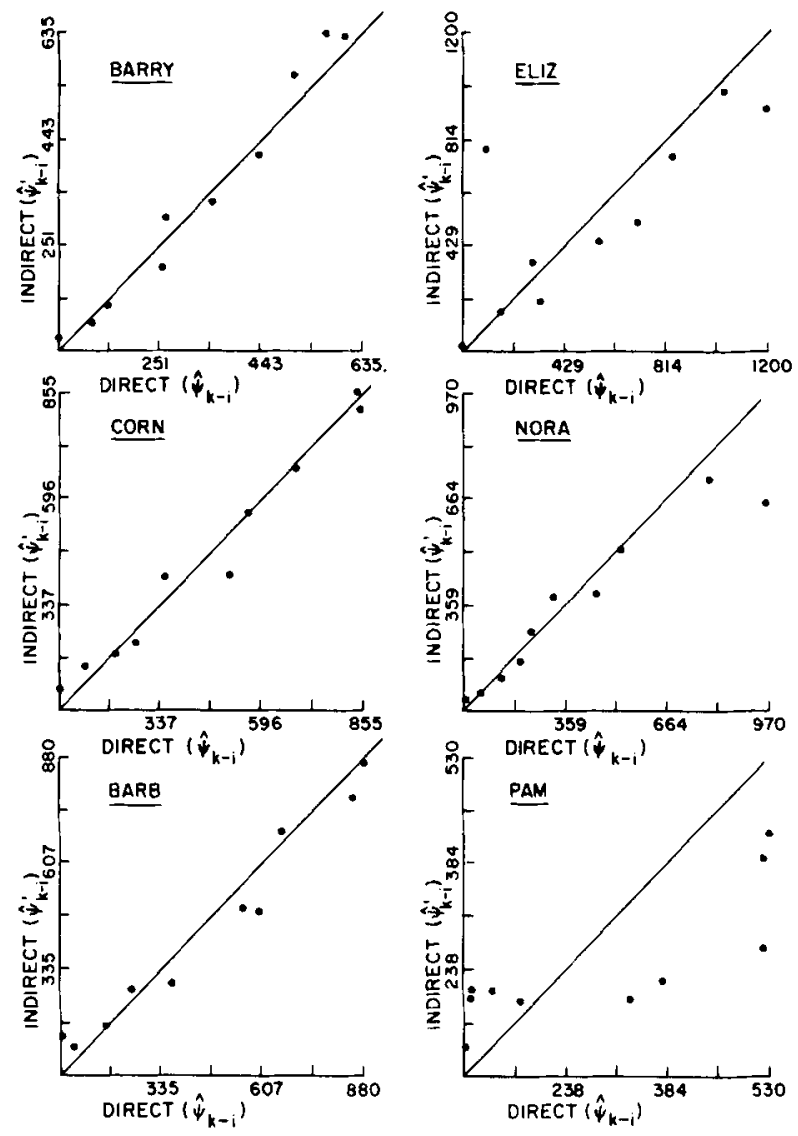

Fig. 2. Test of additive axiom: Plots of mean indirect estimates $\left(\hat{\Psi}_{k-i}^{\prime}\right.$ $\left.=\hat{\Psi}_{k-j}+\hat{\Psi}_{j-j}\right)$ as a function of mean direct estimates $\left(\hat{\Psi}_{k-i}\right)$ based on independent triples. Ss on left did ratio task first and Ss on right did difference task first.

the maximum number (4) of the smallest level $(\mathrm{k}-\mathrm{i}=2)$; (b) the subset should contain at least one triple at all levels except $\mathrm{k}-\mathrm{i}=3$ (Criterion ( 1 ) rules out this level); (c) no other subset should exist for which there are a larger number of independent triples. It can be shown that the maximum number of independent triples is 10 , and the particular subset of 10 selected is the only subset of 10 satisfying the above criteria. Thus, the additive analysis was performed on a set of 10 independent triples and is comparable to the product analysis which involved nine independent triples.

The adequacy of the additive axiom is depicted graphically in Fig. 2, which shows a plot (for each $S$ ) of mean indirect estimates $\left(\hat{\Psi}_{k-j}+\dot{\Psi}_{j-i}=\hat{\psi}_{k-i}^{\prime}\right)$ as a function of mean direct estimates $\left(\hat{\Psi}_{k-i}\right)$ based on the 10 independent triples.

A second method used to analyze the additive data consisted of estimates of scale values for the stimuli based on all difference judgments. Each $\mathrm{W}_{\mathrm{i}}(\mathrm{i}=1, \cdots, 9)$ was treated as a parameter and estimated by least squares. Since one arbitrary assignment was already made to the standard pair, a second arbitrary assignment was made to one of the nine stimuli, providing an interval scale for the stimuli (but a ratio scale for the differences).

Figure 3 shows (for each $S$ ) a plot of the means of observed difference judgments $\left(\Psi_{j-i}\right)$ as a function of predicted scale differences based on all the data, whereas the predicted values in Fig. 2 (a direct test of the additive axiom) are based on only a subset of the data. A comparable analysis for the ratio data cannot be carried out since the method of selection of the tribles rules out the possibility of constructing a scale.

Statistical tests for the product and additive axioms are formally equivalent, since the product law can be transformed to an additive function (Eq. 2). Unlike the typical regression problem, however, the present situation involves error in boti variables-the direct and the indirect estimates. The statistical test was developed as follows. For each stimulus triple ( $i, j, k)$, we have $n=10$ direct estimates and $n$ indirect estimates for the pair $(i, k)$, the indirect estimates based on the two pairs ( $i$, $j$ ) and $(j, k)$. Let $x_{i k}, m$ denote the $m^{t h}$ direct estimate for the pair $(i, k)$ and $y_{i k}, m$ the $m^{\text {th }}$ indirect estimate. Note that in the additive analysis $x$ and $y$ are observed differences whereas in the product analysis $x$ and $y$ are $\log$ ratios. Otherwise the analysis is identical. We assume for each stimulus triple that $\left(x_{i k}, m, y_{i k, m}\right)$ is bivariate normal with mean vector $\left(u_{i k}, v_{i k}\right)$ and common covariance matrix for each $(i, k)$. We wish to test the hypothesis: $\mathrm{H}_{0}: u_{i k}=v_{i k}$ for all $\mathrm{i}, \mathrm{k}(\mathrm{i}<\mathrm{k})$.

Since each observation was associated with a specific (i, k), the error variance was directly estimated from the with in sums of squares and cross products. The deviations from theory are estimated by

$$
\sum_{i k, m}\left(y_{i k, m}-x_{i k, m}\right)^{2} .
$$

If $\mathrm{H}_{0}$ is true, the ratio of the deviations mean square to the within mean square is distributed as F.

Using this test, the product and additive axioms were tested separately for each $S$ and the results are presented in Table 1. Using the .05 level of significance, the product axiom is rejected for all Ss, whereas the fit of the additive axiom is acceptable for two of the Ss.

The last column of Table 1 gives the ratios of the standard error of the best fitting straight line to the ratio data relative
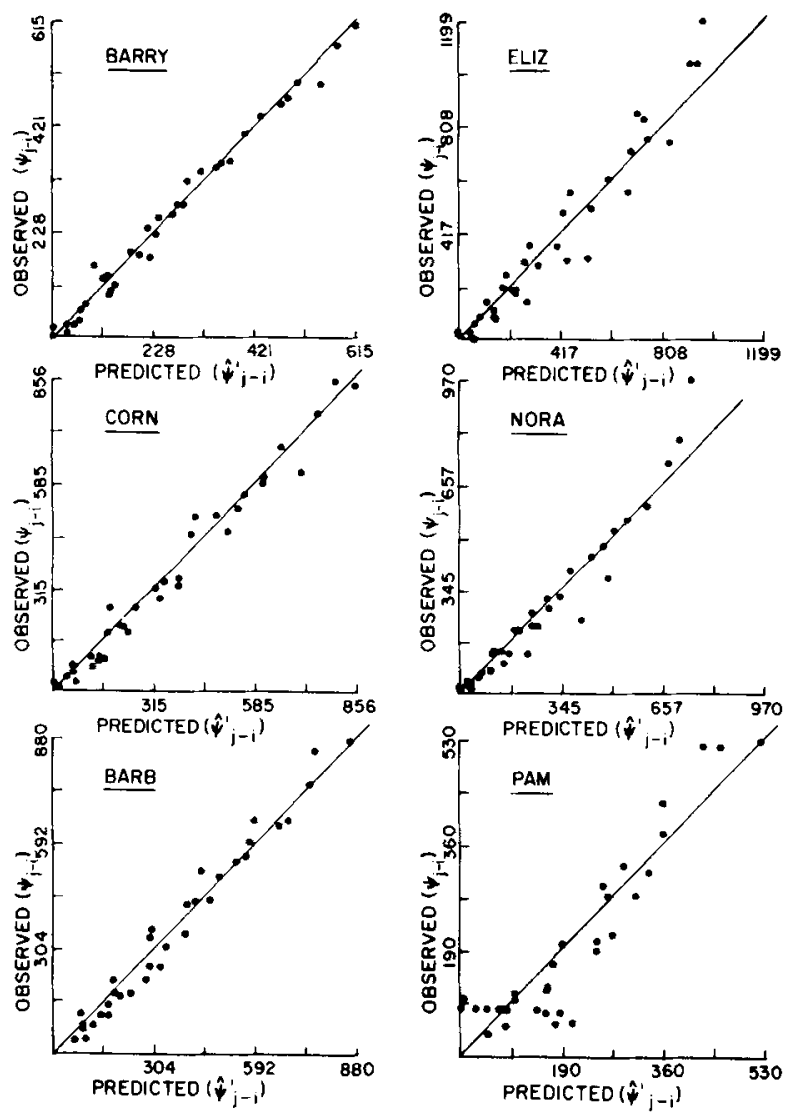

Fig. 3. Test of additive axiom: Plots of means of observed differences $\left(\Psi_{j-i}\right)$ as a function of predicted scale differences $\left(\dot{\Psi}_{j-i}^{\prime}\right)$ based on all data. Ss on left did ratio task first and $S s$ on right did difference task first. 
Table 1

Test of Product and Additive Axioms

\begin{tabular}{lccc}
\hline Subject & $\begin{array}{c}F^{\mathrm{a}} \\
\text { Product }\end{array}$ & $\begin{array}{c}\mathrm{F}^{\mathrm{b}} \\
\text { Additive }\end{array}$ & $\begin{array}{c}\text { Ratio of Standard } \\
\text { Errors of Slope: Product } \\
\text { To Additive }\left(\sigma_{\mathrm{P}} / \sigma_{\mathrm{A}}\right)\end{array}$ \\
\hline Barry $^{\mathrm{T}}$ & $2.90^{* *}$ & 1.41 & 3.34 \\
Corn $^{\mathrm{T}}$ & $2.60^{*}$ & 0.73 & 3.14 \\
Barb $^{\mathrm{I}}$ & $2.17^{*}$ & $2.79^{* *}$ & 2.25 \\
Eliz $^{\mathrm{d}}$ & $7.37^{* * *}$ & $7.50^{* * *}$ & 2.53 \\
Nora $^{\mathrm{d}}$ & $4.74^{* * *}$ & $2.63^{*}$ & 1.21 \\
Pam $^{\mathrm{d}}$ & $12.86^{* * *}$ & $11.56^{* * *}$ & 0.88 \\
\hline
\end{tabular}

$a_{\text {Based on } 8 \& 8 I d f}$

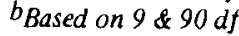

rDid ratio task first

$d_{\text {Did difference task first }}$

* Significant at .05 level

* Significant at .01 level

*** Significant at .0005 level

to the same measure for the difference data. Values greater than 1 mean the standard error was greater for the ratio data. We see that the standard error of the slope was much larger for the ratio data for four of the six Ss, and smaller only for the worst $S$ on both tasks. This is further support for the additive axiom relative to the product axiom.

Figures 1 and 2 , as well as the statistical tests summarized in Table 1, give greater support to the additive axiom relative to the product axiom. The plots shown in Fig. 3, in particular, are encouraging evidence of the tenability of the additive axiom.

Inspection of Fig. 2 suggests a systematic deviation from the additive axiom for those $S$ s rejected by the $F$ test: The direct estimates tend to be too large at the high end of the scale for Eliz, Nora, Pam, and to a lesser extent Barb, which implies that Ss tend to overestimate the larger intervals. However, it would appear from inspection of Fig. 1 that rejection of the product axiom is not a consequence of any obvious systematic deviations.

The relationship between goodness-of-fit to the axioms as judged by inspection of Figs. 1, 2, and 3 and by the F tests deserves some exploration. It is quite possible for the plots to suggest a result inconsistent with the $F$ tests, since the former exhibits only deviations from hypothesis, whereas the $F$ test takes account of error variance as well. It is worth asking, therefore, whether the nonsignificant Fs in Table 1 were bought at the expense of larger error variance, implying greater inconsistency of responses on the part of such Ss. Table 2 lists consistency measures, i.e., $\hat{\rho}=1-\left(\mathrm{MS}_{\mathrm{w} \text { ithin }}\right) /\left(\mathrm{MS}_{\text {total }}\right)$ for each $S$ and each method.

$\hat{\rho}$ (a correlation index with range $0 \leqslant \rho \leqslant 1$ ) is simply a measure of the consistency of response. Although we have available no statistical machinery to draw definite conclusions from this information, it would appear that (a) most Ss tend to be somewhat more consistent making difference judgments, and (b) there is no evidence to indicate that low Fs are being purchased at the expense of greater variability of response.
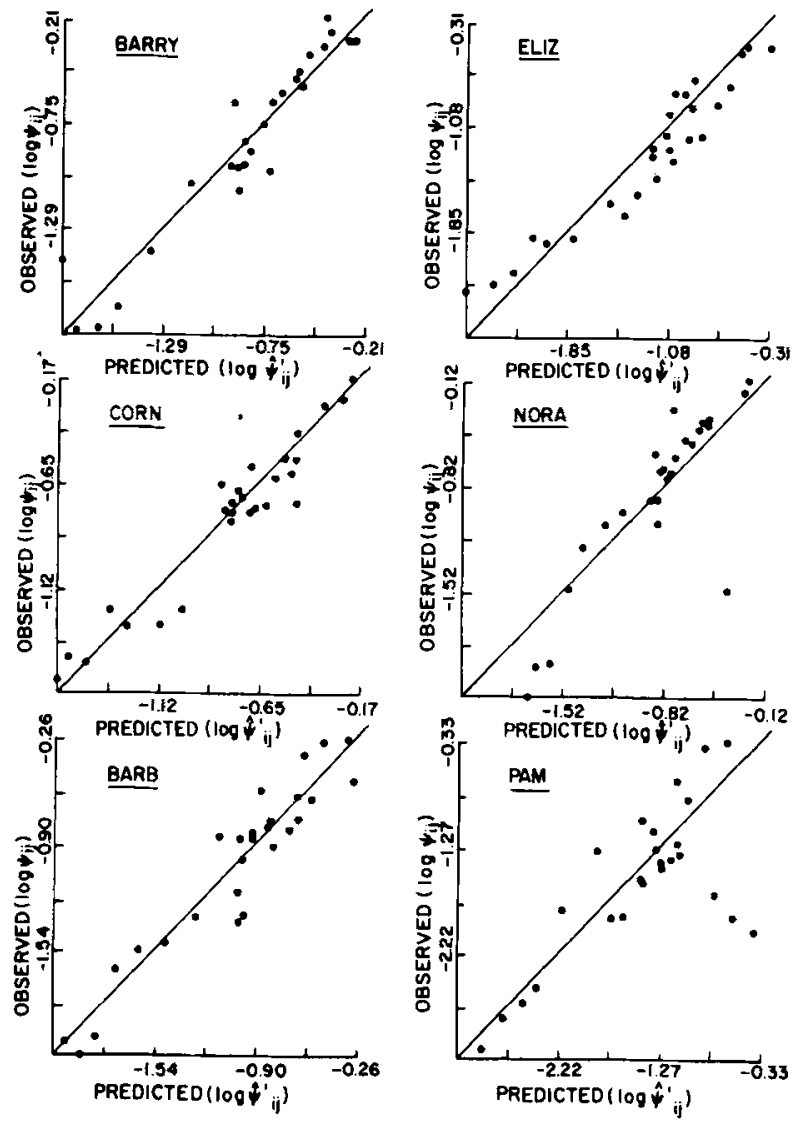

Fig. 4. $\log$ of observed ratios $\left(\log \Psi_{\mathrm{ij}}\right)$ plotted as a function of $\log$ ratios $\left(\log \hat{\Psi}_{i j}^{\prime}\right)$ predicted from simple power law. Ss on left did ratio task first and Ss on right did difference task first.

Although there are too few Ss to devise an adequate statistical test, Table 1 suggests the possible existence of an order effect: Ss who performed the ratio task first (Barty, Corn, Barb) tend to conform more closely to both the product and additive axioms than do Ss who performed the difference task first. In addition, Table 2 indicates that the difference judgments of Ss who did the ratio task first were less variable than for Ss who performed the tasks in reverse order, although no such effect is evident for the ratio task. This order effect is somewhat puzzling in view of the fact that Ss in general conform more closely to the additive axiom.

Psychophysical functions. Three power functions were fitted to the ratio judgment data: the simple power law without additive constant; the $\Psi$-Law, which introduces a threshold parameter via translation on the psychological axis; and the $\Phi$-Law, which introduces a threshold parameter but via translation on the intensity axis (Fagot \& Stewart, 1968).

Least squares iterative procedures were applied to solve for
Table 2

Consistency Measures: $\hat{\rho}=1-\frac{\text { MS }_{\text {Within }}}{\text { MS }_{\text {Total }}}$

\begin{tabular}{lcc}
\hline & \multicolumn{2}{c}{ Type Judgment } \\
\cline { 2 - 3 } Subject & Difference & Ratio \\
\hline Barry & .87 & .69 \\
Corn & .83 & .76 \\
Barb & .84 & .78 \\
Eliz & .83 & .84 \\
Nora & .62 & .77 \\
Pam & .76 & .70 \\
\hline
\end{tabular}

Table 3

Parameter Estimates and F-Tests of Simple Power Function Based on Ratio Judgments

\begin{tabular}{lrr}
\hline Subject & k & Fa, b \\
\hline Barry & .211 & 2.52 \\
Corn & .246 & 2.70 \\
Barb & .276 & 4.00 \\
Eliz & .442 & 7.21 \\
Nora & .271 & 4.70 \\
Pam & .535 & 11.72 \\
\hline
\end{tabular}

Based on 25 and 243 df

$b$ All Fs significant at .0005 level 
Table 4

Parameter Estimates and F-Tests for Psychophysical Functions

Based on Difference Judgments

\begin{tabular}{|c|c|c|c|c|c|c|c|c|}
\hline \multirow[b]{2}{*}{ Subject } & \multicolumn{3}{|c|}{ General Law } & \multicolumn{2}{|c|}{$\Psi$-Law } & \multicolumn{3}{|c|}{ Two-Stage Model } \\
\hline & k & $\alpha$ & $\mathrm{Fa}^{\mathrm{a}}$ & $k$ & $\mathrm{Fb}^{\mathrm{b}}$ & $\mathrm{n}$ & $\mathrm{m}$ & $\mathrm{Fa}$ \\
\hline Barry & .020 & -.2026 & 1.37 & .079 & $1.62 *$ & .086 & $1.01 \mathrm{I}$ & $1.64^{*}$ \\
\hline Corn & -.226 & -.5551 & $4.35 * * *$ & -.057 & $5.22 * * *$ & .048 & 1.182 & $2.32 * * *$ \\
\hline Barb & -.162 & -.3758 & $10.42 * * *$ & .036 & $10.65^{* * *}$ & .122 & 1.278 & $1.98 * *$ \\
\hline Eliz' & -.468 & -.0137 & $7.86 * * *$ & -.122 & $10.20 * * *$ & .073 & 1.361 & $1.51^{*}$ \\
\hline Nora & -.021 & -.0014 & 1.10 & -.020 & 1.07 & .048 & 1.116 & 0.65 \\
\hline \multirow[t]{2}{*}{ Pam } & .043 & -.0156 & $4.51 * * *$ & .206 & $5.20 * * *$ & .184 & 0.948 & $4.93^{* * *}$ \\
\hline & $\begin{array}{l}a \text { Basec } \\
b \text { Basec }\end{array}$ & $\begin{array}{l}\text { on } 33 \\
\text { on } 34\end{array}$ & $\begin{array}{ll}\& & 324 d f \\
\& & 324 d f\end{array}$ & & $\begin{array}{ll}* & \text { Signi } \\
* * & \text { Signi } \\
* * * & \text { Signi }\end{array}$ & $\begin{array}{ll}\text { ant } & 0 \\
\text { ant } & 0 \\
\text { ant } & 0\end{array}$ & $\begin{array}{l}05 \text { leve } \\
01 \text { leve } \\
0005 \text { le }\end{array}$ & \\
\hline
\end{tabular}

the exponent $(\mathrm{k})$ of the simple power law, and jointly for the exponent and threshold parameter ( $t$ ) for the $\Psi$-Law and the $\Phi$-Law. An F test was then used to test for the significance of the deviations from each of the theoretically best-fitting power functions. Estimates of $k$ and $F$ values for a test of the simple power law for each $S$ are presented in Table 3. Note that the simple power law is rejected for all six Ss. Figure 4 shows log of observed ratios $\left(\log \Psi_{\mathrm{ij}}\right)$ plotted as a function of estimates predicted from the simple power law. The deviations from prediction do not appear to be systematic.

Although recent data (Stewart, Fagot, \& Eskildsen, 1967; Fagot \& Stewart, 1968; Irwin \& Corballis, 1968) suggest that the $\Psi$-Law provides a better fit to data than the $\Phi$-Law, no such conclusion can be reached from these data. In fact, neither law provided a noticeably better fit than the simple power law, for which reason parameter estimates, $F$ tests, and plots are omitted. Furthermore, the estimates of $t$ for the $\Phi$-Law were not interpretable as threshold parameters for any of the Ss; three of the Ss produced estimates which were negative, and the other three had estimates which were much too high for this brightness apparatus (Fagot \& Stewart, 1968). The $\Psi$-Law fared somewhat better with respect to this parameter; four of the Ss produced estimates of $t$ which were of the right order of magnitude.

Inability to choose between the $\Psi$-Law and the $\Phi$-Law based on these data is not too surprising, since optimal conditions for such a choice would require a range of stimuli near the threshold, a requirement not adequately met in this experiment. Unfortunately, the requirement of a large range of stimuli for an adequate test of the product axiom was in conflict with the optimal range for discriminating between the special laws (at least with our apparatus).

The psychophysical functions for the difference judgments can be written in the form of the following equations:

$$
\begin{gathered}
\Psi_{j-i}=100\left[\frac{\Phi_{i}^{\mathrm{k}}-\Phi_{\mathrm{j}}^{\mathrm{k}}}{100^{\mathrm{k}}-30^{\mathrm{k}}}\right] \quad \Psi \text {-Law } \\
\Psi_{j-i}=100\left[\frac{\left(\Phi_{\mathrm{i}}-\mathrm{t}\right)^{\mathrm{k}}-\left(\Phi_{\mathrm{j}}-\mathrm{t}\right)^{\mathrm{k}}}{(100-\mathrm{t})^{\mathrm{k}}-(30-\mathrm{t})^{\mathrm{k}}}\right] \quad \text { Q-Law }
\end{gathered}
$$

where $\Psi_{j-i}$ is the observed difference in brightness for stimuli $\left(\Phi_{i}, \Phi_{j}\right)$ relative to the standard pair $(30,100)$. Note that the $\Psi$-Law implies that the difference judgments are independent of the threshold parameter $t$, whereas the $\Phi$-Law implies that the difference judgments are dependent on $\mathrm{t}$.
Inasmuch as the $\Phi$-Law contains two parameters whereas the $\Psi$-Law contains only one parameter, the basis for comparing the two laws is an interesting question. If we assume a more general power function (Fagot, 1966),

$$
\Psi_{i}=a\left(\Phi_{i}-\alpha\right)^{k}+b
$$

for which the $\Psi$-Law and $\Phi$-Law are special cases, then the difference function for the general law is equivalent to Eq. 5 with the substitution $t=\alpha$. The additional parameter implies that the general law must fit better than the $\psi$-Law, although an $F$ test may not show this due to the loss of a degree of freedom in estimating $\alpha$ in the general law. The test we propose to choose between the $\Phi$-Law and the $\psi$-Law hinges on the magnitude of $\hat{t}$. The $\Phi$-Law will be chosen over the $\Psi$-Law only if the magnitude of $\mathrm{t}$ is of the order of magnitude of a threshold parameter.
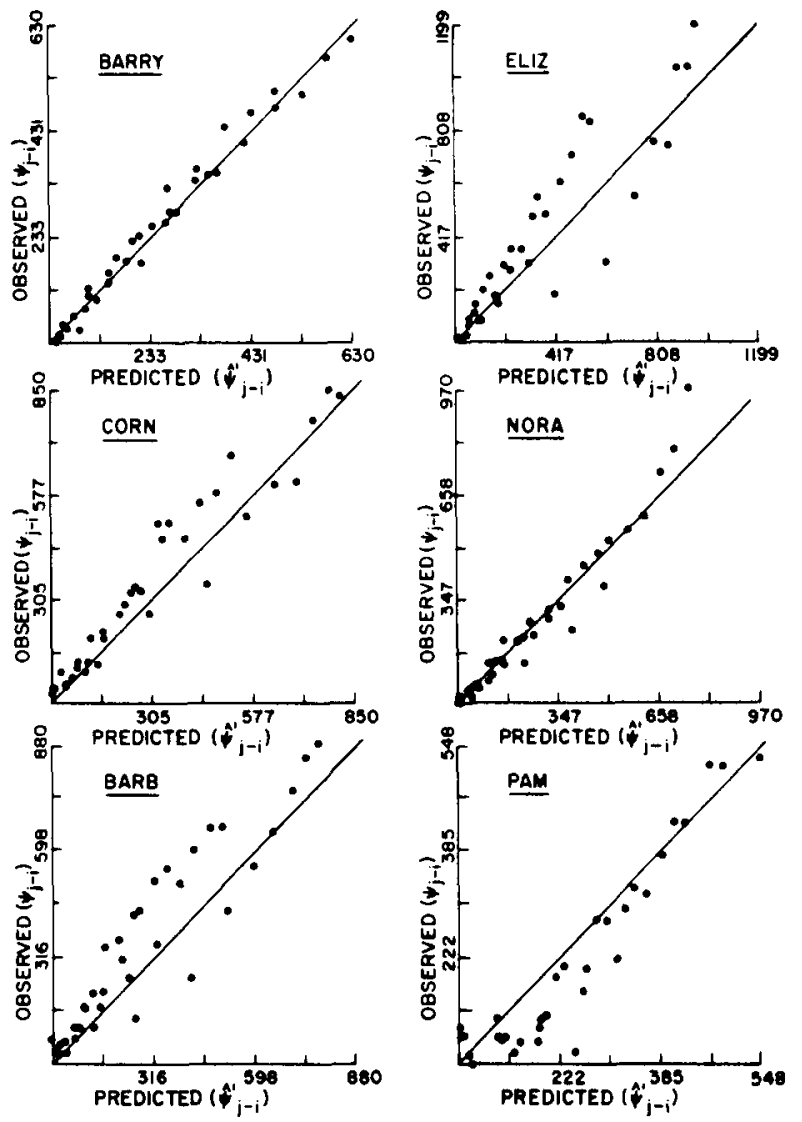

Fig. 5. Mean observed difference estimates $\left(\Psi_{j-i}\right)$ plotted as a function of differences $\left(\hat{\psi}_{j-i}^{\prime}\right)$ predicted from $\Psi$-Law. Ss on left did ratio task first and Ss on right did difference task first. 

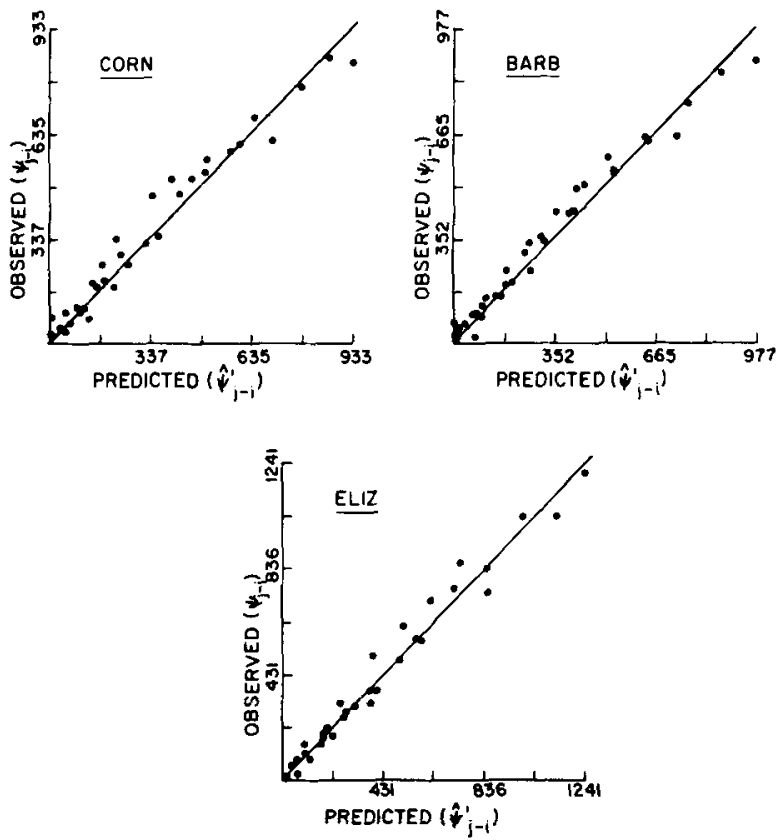

Fig. 6. Mean observed difference estimates $\left(\Psi_{j-i}\right)$ plotted as a function of differences $\left(\hat{\Psi}_{j-i}^{\prime}\right)$ predicted from two-stage model.

Least squares iterative solutions provided (for each $S$ ) estimates of $\mathrm{k}$ for the $\Psi$-Law, and joint estimates of $\mathrm{k}$ and $\alpha$ for the general law. Table 4 presents the parameter estimates and $F$ tests for the psychophysical functions based on difference judgments. The general law was rejected for four of the six $\mathrm{Ss}$, and the $\Psi$-Law was rejected for five of the six Ss, although Barry, one of the two Ss conforming to the general law, was barely significant at the .05 level. Note that $\dot{\alpha}$ was negative for all $S s$ and hence cannot be interpreted as a threshold parameter, suggesting rejection of the $\Phi$-Law.

Figure 5 shows (for each $S$ ) mean observed difference estimates $\left(\Psi_{j-i}\right)$ plotted as a function of estimates predicted from the $\Psi$-Law. Consistent with the $F$ tests, the plots for Barry and Nora are appreciably better than for the other four Ss, for whom the deviations from the $\Psi$-Law do not appear to be systematic.

Curtis, Attneave, and Harrington (1968) have postulated a two-stage model of magnitude estimation which asserts that the psychophysical function for difference judgments is of the form

$$
\Psi_{j-i}=a\left(\Phi_{j}^{n}-\Phi_{i}^{n}\right)^{m}
$$

where $n$ is a sensory (input) exponent and $m$ is an output exponent describing the S's number behavior.

Since four of the Ss provided such poor fits to the difference data, the adequacy of the two-stage model was

Table 5

Test of Two-Stage Additive Axiom

\begin{tabular}{ll}
\hline Subjects & \multicolumn{1}{c}{$\mathrm{F}^{\mathrm{a}}$} \\
\hline Barry & 1.52 \\
Corn & 1.81 \\
Barb & $6.12^{* * *}$ \\
Eliz & $2.77^{* *}$ \\
Nora & 1.87 \\
Parn & $12.36^{* * *}$ \\
\hline
\end{tabular}

a Based on $9 \& 90 d f$

** Significant at .01 level

*** Significant at .0005 level investigated. A least-squares procedure provided joint estimates of $n$ and $m$, and the $F$ test was used to determine goodness-of-fit. These results also are presented in Table 4, and show that three of the four poor Ss (Corn, Barb, Eliz) had substantially reduced Fs although all three Ss still showed significant deviations at least at the .05 level. Figure 6 shows plots of mean observed difference judgments as a function of differences predicted by the two-stage model for the three improved Ss. Comparison of these plots with those for the same $3 \mathrm{Ss}$ in Fig. 5 shows noticeable improvement.

The two-stage model also implies that

$$
\Psi_{\mathrm{k}-\mathrm{i}}^{1 / \mathrm{m}}=\Psi_{\mathrm{k}-\mathrm{j}}^{1 / \mathrm{m}}+\Psi_{\mathrm{j}-\mathrm{i}}^{1 / \mathrm{m}}
$$

and hence that the additive axiom (Eq. 3) holds if and only if $\mathrm{m}=1$. The next step was to test to determine if Eq. 8 gave an appreciably better fit to the difference data than Eq. 3 . Table 5 presents the results of this analysis, and shows that in general the fit of Eq. 8 was better, but not dramatically so.

An additional implication of the two-stage model is a product theorem, which states that $\mathrm{n} \mathrm{m}=\mathrm{k}$, where $\mathrm{k}$ is the exponent determined from a magnitude or ratio estimation experiment. This theorem was confirmed for lifted weights by Curtis, Attneave, and Harrington (1968). To test this implication on our data, we compared the products $\mathrm{n} \mathrm{m}$ determined from the difference judgments with the exponents $\mathrm{k}$ determined from the ratio judgments. The results are presented in Table 6 and show no correspondence whatsoever. Although some discrepancies might be expected due to the

Table 6

Test of Product Theorem $(k=n m)$ for Two-Stage Model

\begin{tabular}{lll}
\hline Subject & $\mathrm{k}$ & $\mathrm{nm}$ \\
\hline Barry & .211 & .087 \\
Corn & .246 & .057 \\
Barb & .276 & .156 \\
Eliz & .442 & .099 \\
Nora & .271 & .054 \\
Pam & .535 & .174 \\
\hline
\end{tabular}

poorly fitting power functions, it would appear that the lack of correspondence is too extreme to be accounted for in this way.

\section{SUMMARY DISCUSSION}

Two previous studies are largely consistent with our results for the product axiom. Mashhour (1961) studied the internal consistency of ratio matrices for Ss making velocity judgments. He did not directly test the product axiom, but first constructed a scale based on all the data, and concluded that the ratio matrix (averaged over $10 \mathrm{Ss}$ ) was not consistent. Presumably a direct test of the product axiom would have led to its rejection. Svenson and $\AA$ kesson (1967) noted systematic departures from the product axiom for Ss judging length of lines, area of circles, and darkness of circles.

In Beck's (1967) study, in which he applied the method of ratio estimation to the judgment of loudness intervals, he reported a better fit to the additive axiom after applying a $\log$ transformation to the difference estimates. It is clear from the application of the two-stage model to our data that such a log transformation would not fit as well as the untransformed additive axiom, and hence his results with loudness would appear to be inconsistent with our results with brightness, a lack of correspondence for which we have no explanation.

Since the power law (of any form) implies the product axiom for ratio judgments and the additive axiom for difference judgments, it is possible that the rejection of the power law is due to lack of conformity to the product or 
additive axiom. An unconfounded test of the power law requires satisfaction of the axioms on which the scale is based. Since the product axiom is rejected for all Ss, it is not surprising that the power law based on ratio data is also rejected for all $\mathrm{Ss}$, and at a lower $\mathrm{p}$ level of significance on the average. If we use the $F$ values as a measure of fit, there is expected correspondence, since the three Ss (Barry, Corn, Barb) who conformed best to the product axiom also gave a better fit to the power function based on ratio data. A similar situation prevails with the difference data, although Corn exhibits the fact that conformity to the additive axiom is no guarantee of conformity to the power function based on difference data.

The results of the tests of the power functions appear more negative than in previous studies. However, it has not been a common practice to perform statistical tests of goodness-of-fit to theoretical power functions. Such statistical analyses might have led to less enthusiasm for the validity of the power function. Nevertheless, some questions could be raised regarding the adequacy of such statistical tests in evaluating "theories" such as the product and additive axioms and the power function, both because of power considerations as pointed out by Fagot and Stewart (1968) and because variability of response favors acceptance of the theory. Fortunately, in this study and in the study by Fagot and Stewart (1968), in which they compared different forms of the power function, variability of response tended to be less for the "good" Ss.

The fact that some Ss conform well to hypotheses like the additive axiom and the power function may be interpreted as encouraging, in spite of the number of Ss who do not fit well. It leaves some hope that violations may be due to inattention, adoption of inappropriate cognitive strategies, etc., and that an adequate theory for "motivated and practiced" observers may be feasible. On the other hand, systematic deviations, such as those apparently present for some of the Ss in the case of the additive axiom (see Fig. 2) may call for the elaboration of the model by introduction of response bias parameters, an approach taken by Sjöberg (1965).

In conclusion, we interpret our data as calling for more attention to scale validity prior to consideration of psychophysical functions, and more strongly for the possibility of a theory of brightness estimation based on an additive axiom for difference judgments than a product axiom for ratio judgments.

\section{REFERENCES}

BECK, J. Ratio-estimations of loudness-intervals. American Journal of Psychology, 1967, 80, 59-65.

CURTIS, D. W., ATTNEAVE, F., \& HARRINGTON, T. L. A test of a two-stage model of magnitude judgment. Perception \& Psychophysics, $1968,3,25-31$.

ESKILDSEN, P. R. An apparatus for studies of brightness which controls variation of intensity with time. American Jounal of Psychology, $1963,76,321-323$.

FAGOT, R. F. Alternative power laws for ratio scaling. Psychometrika, 1966, 31, 201-214.

FAGOT, R. F., \& STEWART, M. R. An experimental comparison of stimulus and response translated power functions for brightness. Perception \& Psychophysics, 1968, 3, 297-305.

IRWIN, R. J., \& CORBALLIS, M. C. On the general form of Stevens' Law for loudness and softness. Perception \& Psychophysics, 1968, 3, 137-144.

MASHHOUR, M. On the validity of scales derived by ratio and magnitude estimation methods. Report from the Psychology Laboratory, University of Stockholm, 1961, No. 105.

POULTON, E. C. The new psychophysics: Six models for magnitude estimation. Psychological Bulletin, 1968, 69, 1-19.

SJÖBERG, L. On ratio estimation. Report from the Psychology Laboratory, University of Stockholm, 1965, No. 191

STEWART, M. R., \& FAGOT, R. F. Invariance tests for bisection and fractionation scaling. Perception \& Psychophysics, 1967, 2, 323-327.

SVENSON, O., \& AKESSON, C. Å. Fractional and multiple estimates in ratio scaling. Report from the Psychology Laboratory, University of Stockholm, 1966, No. 202.

SVENSON, O., \& ÁKESSON, C. A. A further note on fractional and multiple estimates in ratio scaling. Report from the Psychology Laboratory, University of Stockholm, 1967, No. 224.

\section{NOTES}

1. This research was supported in part by National Science Foundation Grant GB4966, and in part by the Advanced Research Projects Agency of the Department of Defense, and was monitored by the Air Force Office of Scientific Research under Contract No. F44620-67-C-0099.

2. Address: Department of Psychology, University of Oregon, Eugene, Oregon 97403.

(Accepted for publication September 20, 1968.) 